\title{
Hydrolysis of Fluorosilanes. A Theoretical Study
}

\author{
Marek Cypryk \\ Center of Molecular and Macromolecular Studies, Polish Academy of Sciences \\ 90-363 Łódź, Sienkiewicza 112, Poland \\ e-mail: mcypryk@cbmm.lodz.pl
}

\section{Cartesian coordinates of $\mathrm{HF} / 6-31+\mathrm{G}^{*}$ optimized structures of fluorosilanes and of stationary points on the hydrolysis pathway}

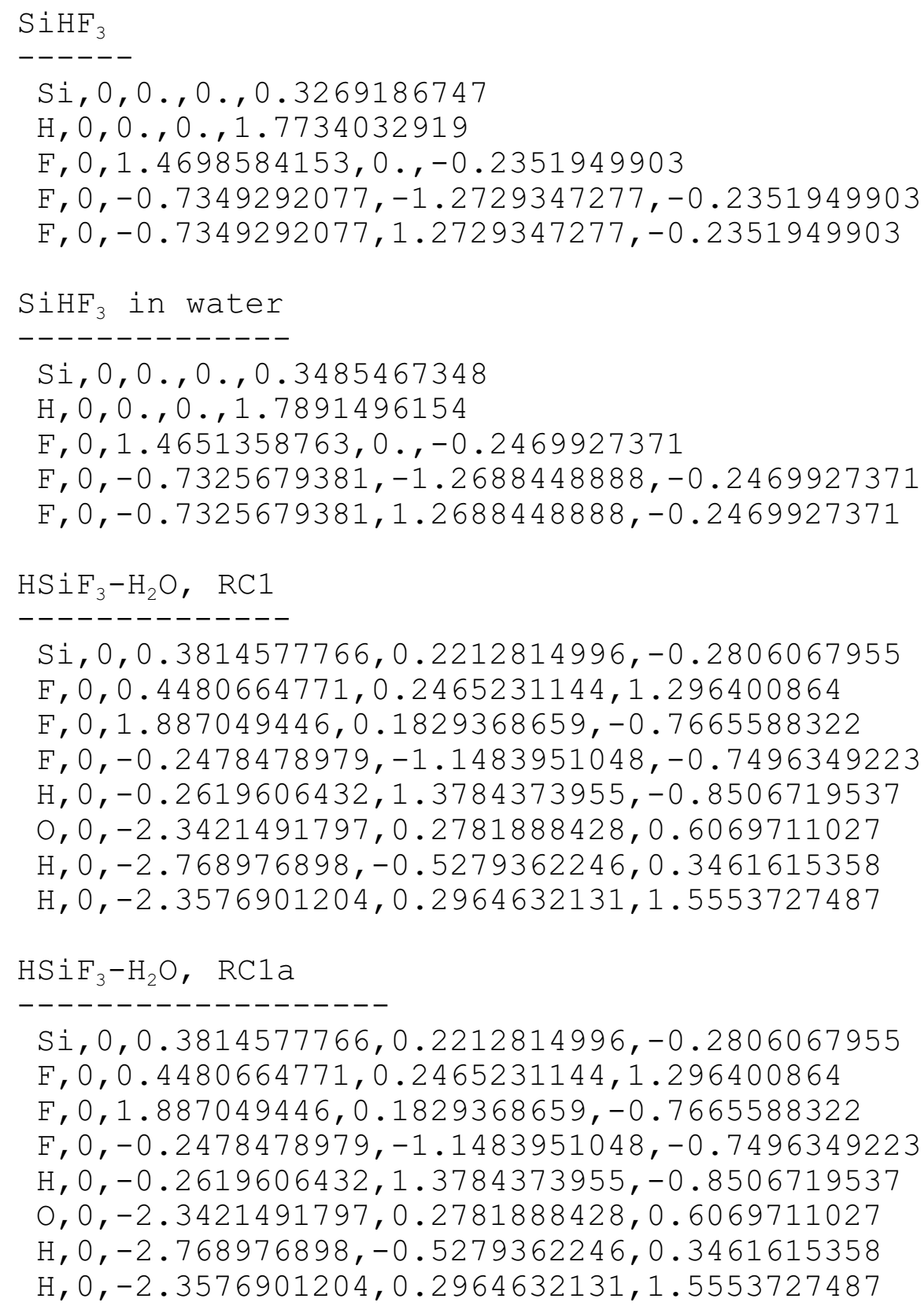


$\mathrm{HSiF}_{3}-\mathrm{H}_{2} \mathrm{O}$, TS

Si, $0,-0.0831775863,0.3900520572,0.1334539005$

$\mathrm{F}, 0,-0.0805149256,0.3773497173,1.743588149$

$\mathrm{F}, 0,1.4779174234,0.3787653954,-0.1242081718$

$\mathrm{F}, 0,-0.3073371003,-0.1347880889,-1.7505571363$

$\mathrm{H}, \mathrm{O},-0.8708570517,1.5599003798,-0.1716257951$

$0,0,-0.8208829505,-1.1991383827,0.0064583585$

$\mathrm{H}, 0,-0.6574413312,-1.0879057324,-1.1179479957$

$\mathrm{H}, \mathrm{O},-0.5507403808,-1.9315596001,0.550146748$

$\mathrm{HSiF} \mathrm{F}_{2} \mathrm{OH}-\mathrm{HF}$, RC2

Si, $0,0.6787391168,0.0141787585,-0.2952580159$

$\mathrm{F}, 0,1.5524442729,1.2740073354,0.0878683541$

$\mathrm{F}, 0,1.575623979,-1.2481577186,0.0200493292$

$\mathrm{F}, 0,-3.2919094956,-0.02464833,-0.2087405309$

$\mathrm{H}, 0,0.2886292866,0.0480206004,-1.687161839$

$0,0,-0.6633002452,-0.0231487704,0.6342541741$

$\mathrm{H}, \mathrm{O},-2.4147710645,-0.0241484025,0.0725393323$

$\mathrm{H}, 0,-0.595232704,-0.0479962345,1.581606966$

$\mathrm{HSiF}{ }_{3}-\mathrm{H}_{2} \mathrm{O}$, RCl in water

Si, 0,-0.0934335662,0.4782553175,0.0025254926

$\mathrm{E}, 0,0.420831108,-0.0712614049,1.3955323612$

$\mathrm{F}, 0,0.4344946691,1.9837251732,-0.0234427262$

$\mathrm{F}, 0,0.7256354367,-0.2235384028,-1.156862733$

$\mathrm{H}, \mathrm{O},-1.5192239824,0.4963604213,-0.1755196466$

$0,0,-1.0441163861,-2.0938058034,0.1356112104$

$\mathrm{H}, \mathrm{O},-1.3658605409,-2.4856237898,-0.6776595584$

$\mathrm{H}, \mathrm{O},-0.428553119,-2.7317230614,0.4998156001$

$\mathrm{HSiF}_{3}-\mathrm{H}_{2} \mathrm{O}$, TS in water

Si, $0,-0.0823415105,0.3959438266,0.212378196$

$\mathrm{F}, 0,-0.057248107,0.4331731053,1.8121634227$

$\mathrm{F}, 0,1.4623357472,0.38437163,-0.1294572059$

$\mathrm{F}, 0,-0.3218795718,-0.2371155829,-1.9264591342$

$\mathrm{H}, 0,-0.8725795899,1.5116746333,-0.2249916978$

$0,0,-0.8093604014,-1.1509109772,0.0033860755$

$\mathrm{H}, \mathrm{O},-0.7028751298,-1.1333131741,-1.062804581$

$\mathrm{H}, \mathrm{O},-0.545753538,-1.938149585,0.4811891881$

$\mathrm{HSiF}_{3}-\left(\mathrm{H}_{2} \mathrm{O}\right)_{2}, \mathrm{RC} 1$

$\begin{array}{llrrr}\mathrm{Si} & 0 & 1.011168 & 0.008209 & -0.262649 \\ \mathrm{~F} & 0 & 1.093420 & 1.090502 & 0.880895 \\ \mathrm{~F} & 0 & 2.357216 & -0.820286 & -0.137704 \\ \mathrm{~F} & 0 & -0.121161 & -1.047080 & 0.085397 \\ \mathrm{H} & 0 & 0.934731 & 0.537756 & -1.599476 \\ \mathrm{O} & 0 & -1.345909 & 1.439557 & -0.526445 \\ \mathrm{H} & 0 & -2.091096 & 0.877021 & -0.325283 \\ \mathrm{H} & 0 & -1.392973 & 2.174981 & 0.069935\end{array}$




$\begin{array}{rrrrr}\mathrm{O} & \mathrm{O} & -3.085110 & -0.714468 & 0.258480 \\ \mathrm{H} & \mathrm{O} & -2.340071 & -1.296013 & 0.355706 \\ \mathrm{H} & \mathrm{O} & -3.784069 & -1.217615 & -0.137366\end{array}$

$\mathrm{HSiF}_{3}-\left(\mathrm{H}_{2} \mathrm{O}\right)_{2}$, TS

Si, $0,0.1552628658,0.7205599293,0.2453248852$

$\mathrm{F}, 0,0.1975157831,1.1190181749,1.8478181998$

$\mathrm{F}, 0,1.7249508887,0.4257074255,0.1345471003$

$\mathrm{F}, 0,-0.0226302462,0.1398669654,-1.4438326287$

$\mathrm{H}, 0,-0.4131585567,1.9877167332,-0.1930752205$

$0,0,-0.8051669349,-0.6638517407,0.6355320631$

$\mathrm{H}, \mathrm{O},-1.0560487336,-1.6856826934,-0.3874040697$

$\mathrm{H}, 0,-0.9225351612,-0.7525300963,1.5735217447$

$0,0,-1.0045716905,-2.0254990019,-1.3622625075$

$\mathrm{H}, 0,-0.5250139199,-1.2071325668,-1.7145811652$

$\mathrm{H}, 0,-1.8775425754,-2.0767375385,-1.7459601693$

$\mathrm{SiHF} \mathrm{OH}_{2} \mathrm{OHF}\left(\mathrm{H}_{2} \mathrm{O}\right), \quad \mathrm{RC} 2$

$\mathrm{Si}, 0,1.1120423001,-0.1942814476,-0.1340997279$

$\mathrm{F}, 0,2.278775896,0.2967804538,-1.0843442023$

$\mathrm{H}, 0,0.2156568838,-1.0854292961,-0.8334199635$

$0,0,0.2794459282,1.1000727971,0.4140256434$

$\mathrm{H}, 0,0.7085948352,1.7306823831,0.9796429861$

$\mathrm{F}, 0,1.8246009003,-0.9070936476,1.0846213083$

$\mathrm{H}, \mathrm{O},-1.7060563147,1.2874655489,0.2697523463$

$0,0,-2.6470507906,1.1564014171,0.1873181922$

$\mathrm{H}, \mathrm{O},-2.9696241719,1.7896212374,-0.4404073843$

$\mathrm{H}, \mathrm{O},-2.9653354421,-0.5404703229,-0.1101303348$

$\mathrm{F}, 0,-2.9823755843,-1.4467671393,-0.3112540109$

$\mathrm{SiHF} \mathrm{H}_{2} \mathrm{OH}$

$\mathrm{Si}, 0,-0.1467354442,0.286308976,-0.1107870442$ $\mathrm{F}, 0,-0.1108393922,0.2414389035,1.4736157489$

$0,0,1.3485351734,0.2133530733,-0.716312927$

$\mathrm{F}, 0,-0.9867315665,-0.9869147587,-0.5422310107$

$\mathrm{H}, \mathrm{O},-0.7969719712,1.49720874,-0.5658444437$

$\mathrm{H}, 0,1.9411254312,-0.5030762935,-0.5350961643$

$\mathrm{SiHF} \mathrm{OH}_{2}$ in water

Si, $0,-0.1386867858,0.292162138,-0.1176434495$

$\mathrm{F}, 0,-0.1166382452,0.2391210552,1.4739657708$

$0,0,1.3508958743,0.220157986,-0.7206245031$

$\mathrm{F}, 0,-0.990213221,-0.9866134208,-0.5372435774$

$\mathrm{H}, \mathrm{O},-0.8052793934,1.496789494,-0.5622123503$

$\mathrm{H}, 0,1.9483117711,-0.5132972523,-0.5328918906$

$\mathrm{HSiF} \mathrm{F}_{2} \mathrm{OH}-\mathrm{H}_{2} \mathrm{O}, \mathrm{RC} 1$

$\mathrm{Si}, 0,0.4773318561,0.0057415955,-0.2787163961$

$\mathrm{F}, 0,-0.0180770107,1.2538509862,0.5733321713$ 
$0,0,2.0985417109,0.0065743572,-0.3134499956$

$\mathrm{F}, 0,-0.0178853749,-1.2762396947,0.5215827841$

$\mathrm{H}, 0,-0.0032212788,0.033522015,-1.6387052143$

$\mathrm{H}, 0,2.6193298391,-0.0095609021,0.4773151211$

$0,0,-2.5379469295,0.0066440718,-0.3352821593$

$\mathrm{H}, \mathrm{O},-2.7298362462,-0.763399441,0.1840247278$

$\mathrm{H}, 0,-2.7300150811,0.754806935,0.2150175508$

$\mathrm{SiHF}_{2}(\mathrm{OH})-\mathrm{H}_{2} \mathrm{O}, \mathrm{RCla}$

Si, $0,0.9417767792,-0.092525938,-0.2496710837$

$\mathrm{F}, 0,0.9564264953,-0.640481198,1.2431654803$

$0,0,-0.2940420002,-0.6958903595,-1.0765248064$

$\mathrm{F}, 0,0.81154108,1.484449483,-0.090871431$

$\mathrm{H}, 0,2.1975369765,-0.4143918735,-0.8987425585$

$\mathrm{H}, \mathrm{O},-1.1894782708,-0.5634324966,-0.7682767269$

$0,0,-2.7341376229,-0.0295292486,0.2499374227$

$\mathrm{H}, \mathrm{O},-2.9835479206,0.8847435912,0.2977152983$

$\mathrm{H}, \mathrm{O},-2.8956568868,-0.4039137904,1.1067517849$

$\mathrm{SiHF} \mathrm{OH}_{2}-\mathrm{H}_{2} \mathrm{O}$, TS

Si, $0,-0.0758255518,0.4052271955,0.1862285288$

$\mathrm{F}, 0,-0.0784021841,0.3859533743,1.7902826634$

$0,0,1.5164989944,0.3724196387,-0.1071351829$

$\mathrm{F}, 0,-0.290016872,-0.1935256538,-1.8120007305$

$\mathrm{H}, 0,-0.9300484908,1.5140942456,-0.1687725016$

$\mathrm{H}, \mathrm{O}, 1.7602126462,0.3186605654,-1.0219364194$

$0,0,-0.9051339961,-1.1524641312,0.0220900122$

$\mathrm{H}, 0,-0.7363886739,-1.0890526901,-1.0590743864$

$\mathrm{H}, \mathrm{O},-0.607366238,-1.9083764024,0.5184078735$

$\mathrm{SiHF}(\mathrm{OH})_{2}-\mathrm{HF}, \mathrm{RC} 2$

$\mathrm{Si}, 0,-0.4035534891,0.7783141402,0.1233402774$

$\mathrm{F}, 0,-0.4940573181,0.8350066279,1.7109538542$

$0,0,1.1122905819,0.8502236185,-0.4128076271$

$0,0,-1.014953009,-0.6941722834,-0.3176718042$

$\mathrm{H}, \mathrm{O},-1.1813208546,1.8924982773,-0.3887828095$

$\mathrm{H}, 0,0.2787514892,-1.8382857367,-0.8960731978$

$\mathrm{H}, 0,1.6291069543,0.0661657032,-0.5608744516$

$\mathrm{H}, 0,-1.8105493987,-1.011138828,0.0905005397$

$\mathrm{F}, 0,1.1557306596,-2.0854564123,-1.0584759112$

$\mathrm{SiHF} \mathrm{FH}_{2}-\mathrm{H}_{2} \mathrm{O}, \mathrm{RC} 1$ in water

Si, $0,0.5219286709,0.0100721989,-0.2657160403$

$\mathrm{F}, 0,0.0338109385,1.2452805936,0.6185254262$

$\mathrm{F}, 0,0.0377188242,-1.2777564339,0.5422305777$

$0,0,2.1348938572,0.0151700005,-0.3672651271$

$\mathrm{H}, \mathrm{O},-0.0445214355,0.0499847754,-1.591999832$

$\mathrm{H}, 0,2.6698105149,-0.0109595826,0.4386904945$

$0,0,-2.7194130268,-0.0034162144,-0.370221224$

$\mathrm{H}, 0,-2.9380616064,-0.7508082247,0.1861770796$ 
$\mathrm{H}, 0,-2.9618433721,0.7690245207,0.1402435945$

$\mathrm{SiHF}{ }_{2} \mathrm{OH}-\mathrm{H}_{2} \mathrm{O}$, TS in water

$\begin{array}{lllll}- & -0.06659 & 0.40784 & 0.25117 \\ \mathrm{~F} & 0 & -0.08326 & 0.43831 & 1.85392 \\ \mathrm{~F} & 0 & -0.34674 & -0.27595 & -2.00905 \\ \mathrm{O} & 0 & 1.50982 & 0.38719 & -0.078 \\ \mathrm{H} & 0 & -0.92535 & 1.46987 & -0.20691 \\ \mathrm{H} & 0 & 1.79264 & 0.37655 & -1.00008 \\ \mathrm{O} & 0 & -0.8562 & -1.12751 & 0.02809 \\ \mathrm{H} & 0 & -0.78127 & -1.15996 & -0.99238 \\ \mathrm{H} & 0 & -0.51271 & -1.93495 & 0.4784\end{array}$

$\mathrm{HSiF} \mathrm{OH}_{2}-\left(\mathrm{H}_{2} \mathrm{O}\right)_{2}, \mathrm{RC} 1$

Si $, 0,-0.1100724273,0.771253368,-0.7383484956$ $\mathrm{F}, 0,-0.0437950663,0.6171403619,0.8513880347$ $\mathrm{H}, \mathrm{O}, 1.1778175833,0.6016890412,-1.3620236449$ $0,0,1.0427672278,-1.9309279029,-0.2658631865$ $\mathrm{H}, 0,1.0631616618,-2.0561351796,0.6797548409$ $\mathrm{H}, 0,0.4481010487,-2.5833597933,-0.6104627944$ $0,0,0.8044014609,-1.6180163978,2.606450284$ $\mathrm{H}, \mathrm{O}, 0.477122817,-0.7451385643,2.4208697999$ $\mathrm{H}, 0,1.4783105232,-1.5282284782,3.2667248195$ $\mathrm{F}, 0,-1.2101934498,-0.26327386,-1.2256205532$ $0,0,-0.6361637504,2.2700336609,-1.0725146713$ $\mathrm{H}, \mathrm{O},-1.5056425145,2.5601124234,-0.8344708251$

$\mathrm{HSi} \mathrm{F}_{2} \mathrm{OH}-\left(\mathrm{H}_{2} \mathrm{O}\right)_{2}$, TS

Si, $0,-0.5795363459,-0.2795660854,0.4314393948$ $\mathrm{F}, 0,-0.5605235021,-0.3773453033,2.0747171349$ $\mathrm{F}, 0,-0.3645973812,-0.1427441524,-1.4188105622$ $\mathrm{H}, 0,-1.0717816534,-1.6289845522,0.1809672548$ $0,0,1.1436487468,-0.0530284189,0.4362344612$ $\mathrm{H}, 0,1.8708912972,0.1064554435,-0.7674249545$ $\mathrm{H}, \mathrm{O}, 1.4849224614,0.0075729322,1.32011777$ $0,0,1.9554686071,0.1612796413,-1.8128731577$ $\mathrm{H}, 0,0.9581689457,0.0613357772,-1.9699894239$ $\mathrm{H}, 0,2.4129523404,-0.6035015084,-2.1540790239$ $0,0,-1.5332249691,1.0538373387,0.3701558238$ $\mathrm{H}, \mathrm{0},-1.7426956779,1.3551437167,-0.501039323$

$\mathrm{SiHF}{ }_{2} \mathrm{OH}+\mathrm{HF}\left(\mathrm{H}_{2} \mathrm{O}\right), \quad \mathrm{RC} 2$

Si, $0,1.1120423001,-0.1942814476,-0.1340997279$

$\mathrm{F}, 0,2.278775896,0.2967804538,-1.0843442023$

$\mathrm{H}, \mathrm{O}, 0.2156568838,-1.0854292961,-0.8334199635$

$0,0,0.2794459282,1.1000727971,0.4140256434$

$\mathrm{H}, \mathrm{O}, 0.7085948352,1.7306823831,0.9796429861$ $\mathrm{F}, 0,1.8246009003,-0.9070936476,1.0846213083$ $\mathrm{H}, \mathrm{O},-1.7060563147,1.2874655489,0.2697523463$ $0,0,-2.6470507906,1.1564014171,0.1873181922$ 
$\mathrm{H}, \mathrm{O},-2.9696241719,1.7896212374,-0.4404073843$

$\mathrm{H}, 0,-2.9653354421,-0.5404703229,-0.1101303348$

$\mathrm{F}, 0,-2.9823755843,-1.4467671393,-0.3112540109$

$\operatorname{SiHF}(\mathrm{OH})_{2}$

Si, $0,-0.1818078049,0.2646430882,-0.1284755939$

$\mathrm{F}, 0,-0.2712483154,0.2794752815,1.4657257591$

$0,0,1.3377090258,0.506839593,-0.6350030469$

$0,0,-0.6659329855,-1.2157772529,-0.6125730452$

$\mathrm{H}, \mathrm{O},-1.012421605,1.3537466348,-0.6146513304$

$\mathrm{H}, 0,1.9525997606,-0.2130047179,-0.6684576122$

$\mathrm{H}, \mathrm{O},-1.3278423709,-1.689521406,-0.1291558374$

$\operatorname{SiHF}(\mathrm{OH})_{2}$ in water

Si, $0,-0.1726302762,0.2308966302,-0.1281369486$ $\mathrm{F}, 0,-0.1771945538,0.3121636249,1.4759758576$

$0,0,1.3219002236,0.5134499985,-0.6866510492$

$0,0,-0.6349011917,-1.2675437785,-0.5551959744$

$\mathrm{H}, 0,-1.0526455753,1.2889081759,-0.5993383193$

$\mathrm{H}, 0,2.0075765212,-0.1570043103,-0.6036788328$

$\mathrm{H}, \mathrm{0},-1.461049443,-1.6344691199,-0.2255654402$

$\mathrm{SiHF}(\mathrm{OH})_{2}-\mathrm{H}_{2} \mathrm{O}, \mathrm{RC} 1$

$\mathrm{Si}, 0,-0.4757258911,0.7685927216,-0.0632521014$

$\mathrm{F}, 0,-1.0352625049,0.9076527808,1.4249529118$

$0,0,1.1310541634,0.788971018,-0.1053834399$

$0,0,-0.9838842124,-0.6963303183,-0.6206004457$

$\mathrm{H}, 0,-1.0374350323,1.8832328501,-0.8119392802$

$\mathrm{H}, 0,1.6106382747,-0.0366848609,-0.1491598926$

$\mathrm{H}, \mathrm{O},-1.9027418414,-0.9196537302,-0.5601437684$

$0,0,1.6797211953,-1.9769985365,-0.4973479025$

$\mathrm{H}, 0,0.7488265037,-2.0708006914,-0.6730036415$

$\mathrm{H}, 0,1.9431079445,-2.7104040037,0.0418541003$

$\mathrm{SiHF}(\mathrm{OH})_{2}-\mathrm{H}_{2} \mathrm{O}, \mathrm{RCla}$

Si, $0,0.6228845793,0.4075035862,-0.5174870312$

$\mathrm{F}, 0,0.266147971,1.5748729133,0.5018816674$

$0,0,1.1921182597,-0.8879440249,0.2647210174$

$0,0,-0.8149693534,0.1072517326,-1.2555798559$

$\mathrm{H}, 0,1.6410368499,0.8733046454,-1.4477583013$

$\mathrm{H}, 0,0.5724540749,-1.3400397108,0.832541246$

$\mathrm{H}, \mathrm{O},-0.8346534408,-0.4401678157,-2.028241018$

$0,0,-1.4731358653,-1.3545218212,1.3249973252$

$\mathrm{H}, 0,-1.8777638145,-0.9106207407,0.5875234734$

$\mathrm{H}, \mathrm{O},-1.8488938475,-0.9796698972,2.110710137$

$\mathrm{SiHF}(\mathrm{OH})_{2}-\mathrm{H}_{2} \mathrm{O}, \mathrm{TS}$

Si, $0,0.0691318307,0.2589877626,0.5542542917$

$\mathrm{H}, 0,0.0017499764,0.3481978232,2.0077304912$ 
$0,0,1.6551302411,0.1074537079,0.2505315884$ $0,0,-0.8586658905,1.4673418223,0.0168426372$ $\mathrm{H}, 0,1.8769813343,-0.0224377271,-0.663930068$ $0,0,-0.7817013752,-1.3403657407,0.3884422883$ $\mathrm{H}, 0,-0.6335894027,-1.3517970175,-0.6327747616$ $\mathrm{H}, 0,-0.4221054872,-2.0932799427,0.8483137957$ $\mathrm{F}, 0,-0.1011204316,-0.4293708734,-1.5158911406$ $\mathrm{H}, \mathrm{O},-0.9989019694,1.4823877315,-0.9224113867$

$\mathrm{SiH}(\mathrm{OH})_{3}-\mathrm{HF}, \mathrm{RC} 2$

$\mathrm{Si}, 0,-0.70884876,0.2347490037,-0.3977357482$ $0,0,-0.7268635219,-0.0452239787,1.2008428677$ $0,0,0.9047296306,0.2214922978,-0.815888503$ $\mathrm{H}, 0,-1.2981014591,1.5078101199,-0.7898988188$ $\mathrm{H}, \mathrm{O}, 2.0350297602,0.2456796968,0.5583252673$ $\mathrm{H}, 0,0.0548151109,0.0718387148,1.7281121931$ $\mathrm{H}, \mathrm{O}, 1.1461603203,0.2693837987,-1.7315871417$ $\mathrm{F}, 0,2.2677345138,0.2652139165,1.4560275421$ $0,0,-1.5375225436,-0.9212988781,-1.1905412317$ $\mathrm{H}, \mathrm{O},-1.5463802378,-1.8078791578,-0.8562039681$

$\mathrm{SiHF}(\mathrm{OH})_{2}-\mathrm{H}_{2} \mathrm{O}$ in water, $\mathrm{RC} 1$

Si, $0,-0.4935606657,0.8323042418,0.2271174778$ $\mathrm{F}, 0,-0.1321872278,0.5343031856,1.7653494696$ $0,0,0.7991758037,0.5657166261,-0.7029812626$ $0,0,-1.695282802,-0.1770454276,-0.2018860504$ $\mathrm{H}, 0,-0.8505217137,2.2415504631,0.1549140285$ $\mathrm{H}, 0,1.1115491525,-0.3280873901,-0.8710100243$ $\mathrm{H}, 0,-2.5076467332,-0.207513618,0.3122935846$ $0,0,1.7543691843,-2.0231351296,-1.1916941102$ $\mathrm{H}, 0,1.1121832751,-2.7160408833,-1.3444323153$ $\mathrm{H}, 0,2.3678729007,-2.3751851796,-0.547063803$

$\mathrm{SiHF}(\mathrm{OH})_{2}-\mathrm{H}_{2} \mathrm{O}$ in water, TS

Si, $0,0.0758631915,0.2424758631,0.5876602676$ $\mathrm{H}, 0,0.0167721505,0.33762759,2.0398102904$ $0,0,1.6559585478,0.1552348444,0.2441963141$ $0,0,-0.852289825,1.4477281125,0.0378063999$ $\mathrm{H}, 0,1.9053030451,0.025271267,-0.672816319$ $0,0,-0.7591537699,-1.3099526175,0.3709080996$ $\mathrm{H}, 0,-0.640226941,-1.4235716228,-0.6207009578$ $\mathrm{H}, 0,-0.5067335111,-2.0898710202,0.8901354577$ $\mathrm{F}, 0,-0.0980419887,-0.4650052073,-1.6363856192$ $\mathrm{H}, 0,-0.9905420733,1.5071803369,-0.9095061976$

$\mathrm{SiHF}(\mathrm{OH})_{2}-\left(\mathrm{H}_{2} \mathrm{O}\right)_{2}, \mathrm{RC} 1$

Si, $0,-0.5732943158,0.2176601069,0.8634302512$ $0,0,-0.5470438082,0.1678121231,2.5009961759$ $\mathrm{F}, 0,0.9539191977,0.4153428552,0.402813918$ $\mathrm{H}, \mathrm{O},-1.3420355357,1.3342567178,0.3560258724$ 
$0,0,-1.1377084425,-1.2406346533,0.4135524451$ $\mathrm{H}, \mathrm{O},-1.2052751343,-1.8990032885,1.0925466966$ $\mathrm{H}, 0,-0.1111742562,0.8376849988,3.0074550458$ $0,0,-0.7318007192,0.1432972244,-2.1190598105$ $\mathrm{H}, \mathrm{0}, 0.1668550226,0.0623282931,-2.426322474$ $\mathrm{H}, \mathrm{O},-1.0847435741,-0.7361834146,-2.0879487887$ $0,0,2.1819362786,0.0233102676,-2.2586702774$ $\mathrm{H}, 0,2.1279797944,0.1921719403,-1.3245204193$ $\mathrm{H}, 0,2.7661748546,0.6731378653,-2.6251329774$

$\operatorname{SiHF}(\mathrm{OH})_{2}-\left(\mathrm{H}_{2} \mathrm{O}\right)_{2}$, TS

Si, $0,0.4092366894,-0.4414015209,0.4749946864$ $0,0,0.3071228855,-0.3646643336,2.1643324903$ $\mathrm{H}, 0,1.8566361646,-0.4424928055,0.282797883$ $0,0,-0.3266829041,1.1333042231,0.3725558583$ $\mathrm{H}, 0,-0.5769563292,1.4372282981,1.2374261737$ $0,0,-0.4813886949,-1.8236956952,0.3145307256$ $\mathrm{H}, 0,-0.5933872969,-2.1210127586,-0.5764170277$ $\mathrm{H}, \mathrm{O}, 0.0688255472,-1.1753850416,2.5904460939$ $\mathrm{F}, 0,0.3149464782,-0.3142914149,-1.4276092003$ $\mathrm{H}, 0,-0.5701681562,1.7496967044,-0.8237373426$ $0,0,-0.5758879421,1.8291754577,-1.8903598107$ $\mathrm{H}, 0,-0.1970945079,0.8942921785,-2.0158117264$ $\mathrm{H}, 0,0.0630058684,2.472960235,-2.184620969$

$\mathrm{SiH}(\mathrm{OH})_{3}-\mathrm{HF}\left(\mathrm{H}_{2} \mathrm{O}\right), \quad \mathrm{RC} 2$

$\mathrm{Si}, 0,-0.5616470235,0.9605214006,0.2324112334$ $\mathrm{H}, 0,-0.5150717928,0.8477709395,1.6865728004$ $0,0,1.0245278746,0.9227578187,-0.2465887579$ $\mathrm{H}, \mathrm{O}, 1.2345866126,1.3791864893,-1.0520034657$ $\mathrm{H}, 0,2.0832964658,-0.666261942,0.0896483995$ $0,0,2.2946736229,-1.5894978474,0.2290372101$ $\mathrm{H}, 0,2.8870346943,-1.642693939,0.9674525163$ $\mathrm{H}, 0,0.8172898474,-2.4234328931,0.2159883787$ $\mathrm{F}, 0,-0.0831702341,-2.6794992632,0.1997350381$ $0,0,-1.4548802469,-0.2162804912,-0.444575879$ $\mathrm{H}, \mathrm{O},-1.2318464366,-1.1308903619,-0.2975862333$ $0,0,-1.1899010881,2.3753511616,-0.2874860737$ $\mathrm{H}, 0,-2.0590602537,2.3658703343,-0.6645370021$

$\mathrm{HSi}(\mathrm{OH})_{3}$

$0,0,-0.305527857,0.1318168954,-1.5506635389$ $\mathrm{Si}, 0,-0.3117442296,0.127906622,0.0816625383$ $\mathrm{H}, 0,0.5214422428,0.1227244975,-2.0126199506$ $0,0,0.9056123884,1.069583937,0.6307933667$

$\mathrm{H}, \mathrm{O},-1.6343881506,0.6059169341,0.4721458593$ $0,0,-0.0158350721,-1.3531026469,0.7280345082$ $\mathrm{H}, 0,-0.5911901567,-2.081793724,0.5449643123$ $\mathrm{H}, 0,1.3945596034,0.7760741008,1.3869195547$

$\mathrm{MeSiF}_{3}$ 
Si, 0,0.0179043643,0.0289458065,0.01300959

$\mathrm{F}, 0,0.0324701959,0.0524932455,1.5916366174$

$\mathrm{F}, 0,1.523918368,0.0524716412,-0.4604754013$

$\mathrm{F}, 0,-0.6335485815,1.3869743525,-0.4604746188$

$\mathrm{C}, 0,-0.8876615685,-1.4350669218,-0.6451169525$

$\mathrm{H}, \mathrm{O},-0.4244793479,-2.3573332033,-0.3084731651$

$\mathrm{H}, 0,-1.9196986623,-1.4324689278,-0.3084652953$

$\mathrm{H}, \mathrm{O},-0.8860735213,-1.4324907819,-1.73067346$

$\mathrm{MeSiF}{ }_{3}-\mathrm{H}_{2} \mathrm{O}, \mathrm{RC} 1$

Si, $0,0.491284976,0.032030163,-0.1570875239$

$\mathrm{F}, 0,0.4163601959,0.0405035353,1.4242372525$

$\mathrm{F}, 0,2.0330055233,-0.0934228247,-0.5019279825$

$\mathrm{F}, 0,-0.1596228773,-1.3224589562,-0.6550350728$

$0,0,-2.5020948577,-0.1856618438,0.8148112175$

$\mathrm{H}, \mathrm{0},-2.6676441271,-1.0674668534,0.5072896721$

$\mathrm{H}, \mathrm{0},-2.3250960832,-0.2568872424,1.7438723095$

$\mathrm{C}, 0,-0.2276008942,1.5337863711,-0.9423484396$

$\mathrm{H}, 0,-0.1126520459,1.4886869967,-2.0214002705$

$\mathrm{H}, 0,-1.2812778786,1.6224478808,-0.7085850266$

$\mathrm{H}, 0,0.2833591206,2.4257776705,-0.5918182267$

MeSiF $\mathrm{F}_{3}-\mathrm{H}_{2} \mathrm{O}$, TS

Si, 0,0.0837199911,0.1460507947,0.1600301597

$\mathrm{F}, 0,0.2622836226,0.1731624219,1.7663039858$

$\mathrm{F}, 0,1.6144717358,0.0902905343,-0.2478222878$

$\mathrm{F}, 0,-0.3018564638,-0.5386502419,-1.6876419716$

$0,0,-0.6639558275,-1.4539887838,0.1959594775$

$\mathrm{H}, \mathrm{O},-0.5918881758,-1.4335563381,-0.9269487229$

$\mathrm{H}, \mathrm{O},-0.3075515707,-2.1451344666,0.7443211468$

C, $0,-0.9417594752,1.6378045609,-0.2080720335$

$\mathrm{H}, 0,-0.8165227019,2.3863786981,0.5662577749$

$\mathrm{H}, 0,-0.674326299,2.0601231738,-1.1690619934$

$\mathrm{H}, 0,-1.9936777086,1.3693362828,-0.251791598$

MeSiF $\mathrm{F}_{2} \mathrm{OH}-\mathrm{HF}$, RC2

Si, 0,0.4707592952,0.2592072431,0.3611540328

$\mathrm{F}, 0,0.4713877398,0.2625455302,1.9471927287$

$\mathrm{F}, 0,2.0028279932,0.2625983068,-0.0490683435$

$\mathrm{F}, 0,-2.3619716773,-1.3367681943,-1.8120448607$

$0,0,-0.1962645707,-1.1490825739,-0.1505935101$

$\mathrm{H}, 0,-1.6303065419,-1.3139101575,-1.2507323366$

$\mathrm{H}, 0,0.1726996334,-1.9772712813,0.1324351801$

C, $0,-0.4443200015,1.694995576,-0.3408148117$

$\mathrm{H}, 0,-0.4167121209,1.6802704238,-1.4258116713$

$\mathrm{H}, \mathrm{O},-1.4851341374,1.6802326367,-0.0331051212$

$\mathrm{H}, \mathrm{O},-0.005336892,2.6290833255,-0.0040212787$

$\mathrm{MeSiF}_{3}-\left(\mathrm{H}_{2} \mathrm{O}\right)_{2}, \mathrm{RC} 1$ 
Si, $0,0.8773970276,0.4150733763,-0.079169316$ $\mathrm{F}, 0,0.8124356447,0.3672992349,1.4980110546$ $\mathrm{F}, 0,2.4176903024,0.285681465,-0.4358597227$ $\mathrm{F}, 0,0.2366367848,-0.9310604155,-0.6325509015$ $0,0,-2.1318707693,0.3175760738,0.8190399324$ $\mathrm{H}, \mathrm{O},-2.4246048949,-0.5404230772,0.5217253873$ $\mathrm{H}, 0,-2.1115311028,0.2776272229,1.765680026$ $0,0,-2.3430503269,-2.362012652,-0.2642838476$ $\mathrm{H}, \mathrm{O},-1.4547397828,-2.2928140986,-0.5943549478$ $\mathrm{H}, \mathrm{O},-2.8559979688,-2.8030844173,-0.9279450209$ C, $0,0.1746143182,1.9397106003,-0.8321606785$ $\mathrm{H}, 0,-0.8809777999,2.0258869021,-0.609521535$ $\mathrm{H}, 0,0.6869514014,2.8174281627,-0.4486691015$ $\mathrm{H}, 0,0.3081600356,1.9242985004,-1.9100328647$

$\mathrm{MeSiF}-\left(\mathrm{H}_{2} \mathrm{O}\right)_{2}$, TS

Si, $0,0.14279954,0.4002519254,0.3902795946$ $\mathrm{F}, 0,0.1948197877,0.3319843121,2.0473916347$ $\mathrm{F}, 0,1.7375992919,0.3853453869,0.2216446091$ $\mathrm{F}, 0,0.0318076627,0.1965447653,-1.4113596623$ $0,0,-0.5931043531,-1.170996816,0.4033174606$ $\mathrm{H}, 0,-0.6314504942,-1.9620652474,-0.814831594$ $\mathrm{H}, 0,-0.6564118438,-1.5091236938,1.2880846652$ $0,0,-0.5062986719,-2.0717996877,-1.8395272227$ $\mathrm{H}, 0,-0.1937613599,-1.1164903774,-1.9797298972$ $\mathrm{H}, 0,-1.34923919,-2.2004780014,-2.2684966841$ C, $0,-0.8244820922,1.9924710703,0.2447168935$ $\mathrm{H}, \mathrm{O},-0.4246922523,2.6168564676,-0.5475994241$ $\mathrm{H}, 0,-1.8608012789,1.7813847506,-0.0134638742$ $\mathrm{H}, 0,-0.8187610679,2.5490645751,1.1744099868$

$\mathrm{SiMeF}{ }_{2} \mathrm{OH}-\mathrm{HF}\left(\mathrm{H}_{2} \mathrm{O}\right), \quad \mathrm{RC} 2$

Si, $0,-0.4044196296,0.666914892,0.8921517142$ $\mathrm{F}, 0,-0.3528802965,0.8482706336,2.4694506637$ $0,0,1.1379214753,0.5835894034,0.3400798365$ $\mathrm{H}, 0,1.715528096,1.3224000917,0.489300305$ $\mathrm{F}, 0,-1.0497784495,2.0120128105,0.351703981$ $\mathrm{H}, 0,1.8669366462,-0.6805834139,-0.9835634293$ $0,0,2.0913680053,-1.2852379789,-1.6873127739$ $\mathrm{H}, 0,2.6564354115,-1.9526521172,-1.3211247099$ $\mathrm{H}, 0,0.6638885866,-1.7785087268,-2.5565769484$ $\mathrm{F}, 0,-0.1711324723,-2.0007179179,-2.898766087$ C, $0,-1.3661580391,-0.8252682439,0.4052584534$ $\mathrm{H}, 0,-1.3736699485,-0.9637016213,-0.6708419653$ $\mathrm{H}, \mathrm{0},-0.9460894978,-1.7200474996,0.8547534383$ $\mathrm{H}, 0,-2.3944011258,-0.7350068689,0.7427450716$

$\mathrm{MeSiF} \mathrm{FH}_{2} \mathrm{OH}$

Si, $0,0.0059799024,-0.0221174809,0.0045885048$ $\mathrm{F}, 0,0.0161589513,0.0200303258,1.6235920709$ $\mathrm{F}, 0,1.5723557463,0.0200348382,-0.4049773394$ 
$0,0,-0.6654293028,-1.4286483216,-0.5104855534$

$\mathrm{H}, 0,-0.2974023322,-2.2736538834,-0.2282428847$

C, $0,-0.909864411,1.4174788522,-0.6979800876$

$\mathrm{H}, 0,-0.8776826686,1.394965512,-1.791327431$

$\mathrm{H}, 0,-1.9575363116,1.3949930444,-0.383608939$

$\mathrm{H}, \mathrm{O},-0.4651087107,2.3570670439,-0.3568274426$

$\mathrm{MeSiF} \mathrm{OH}_{2}-\mathrm{H}_{2} \mathrm{O}, \mathrm{RC} 1$

Si, $0,-0.4069941699,0.3807899441,-0.0988649047$

$\mathrm{F}, 0,-0.3152427623,0.3185470329,1.4936762731$

$0,0,1.0707223407,0.4737883809,-0.733731503$

$\mathrm{F}, 0,-1.0813187645,-1.0138218862,-0.4846440875$

$\mathrm{H}, 0,1.6938914641,-0.2149891833,-0.5111937374$

$0,0,2.2411693627,-1.8887088986,0.4042266601$

$\mathrm{H}, 0,1.7712371848,-2.6735768558,0.1514732287$

$\mathrm{H}, 0,2.2364284212,-1.8644840495,1.3529775855$

C, $0,-1.4739973553,1.7901634268,-0.6348676438$

$\mathrm{H}, \mathrm{O},-1.0413855605,2.737926706,-0.3293710575$

$\mathrm{H}, 0,-1.5781865724,1.8043838557,-1.7154594428$

$\mathrm{H}, \mathrm{O},-2.4661623128,1.7155375697,-0.2003629754$

MeSi $\mathrm{F}_{2} \mathrm{OH}-\mathrm{H}_{2} \mathrm{O}$, TS

Si, $0,0.0956670656,0.1793751126,0.2119596031$

$\mathrm{F}, 0,0.2087946889,0.2129504033,1.8171044285$

$0,0,1.6679253659,0.1232054949,-0.1822554413$

$\mathrm{F}, 0,-0.2043853222,-0.6082570664,-1.755053647$

$\mathrm{H}, 0,1.8416279772,-0.0110387459,-1.1048365869$

$0,0,-0.7014068784,-1.417715945,0.1997179914$

$\mathrm{H}, 0,-0.5904811615,-1.4512077596,-0.869628686$

$\mathrm{H}, \mathrm{O},-0.3244051573,-2.1198477806,0.7211180385$

$\mathrm{C}, 0,-1.0352645806,1.570902345,-0.2382698847$

$\mathrm{H}, \mathrm{O},-0.9572852049,2.3675728823,0.4943986016$

$\mathrm{H}, 0,-0.8024268478,1.9612770013,-1.2210532306$

$\mathrm{H}, 0,-2.0666132402,1.2304223242,-0.255970706$

$\operatorname{MeSiF}(\mathrm{OH})_{2}-\mathrm{HF}, \mathrm{RC} 2$

Si, $0,-0.1731909461,0.4839907107,0.2207570676$

$\mathrm{F}, 0,-0.1646986076,0.4358670407,1.8169285541$

$0,0,1.3240622996,0.481237068,-0.3845013972$

$0,0,-0.8788634358,-0.9453601619,-0.2441498791$

$\mathrm{H}, 0,0.3091766221,-2.1244411473,-0.9054727825$

$\mathrm{H}, 0,1.7979233004,-0.3284280497,-0.5347948908$

$\mathrm{H}, \mathrm{O},-1.6199701625,-1.272091264,0.2495559786$

$\mathrm{F}, 0,1.1456202136,-2.4621387955,-1.1163322236$

C, $0,-1.0982591225,1.9933205659,-0.3154649541$

$\mathrm{H}, 0,-0.568660689,2.8911913417,-0.0107011441$

$\mathrm{H}, 0,-1.2027116509,2.0185067368,-1.3956531713$

$\mathrm{H}, \mathrm{0},-2.0914148045,2.028899581,0.1231000243$

$\mathrm{MeSiF}{ }_{2} \mathrm{OH}-\left(\mathrm{H}_{2} \mathrm{O}\right)_{2}, \mathrm{RC} 1$ 
Si, $0,-0.4308067515,0.0847027822,0.8624401641$ $\mathrm{F}, 0,-0.341160592,-0.0269070393,2.4530604454$ $\mathrm{F}, 0,1.1048829481,0.1776206975,0.4223153033$ $0,0,-0.4238769795,-0.0679148567,-2.371272455$ $\mathrm{H}, 0,0.5033319031,-0.2121366105,-2.5389574938$ $\mathrm{H}, \mathrm{O},-0.8044430155,-0.9250816454,-2.2325880212$ $0,0,2.4677001756,-0.4241057866,-2.1344076817$ $\mathrm{H}, 0,2.3832552984,-0.1805019735,-1.2195070639$ $\mathrm{H}, 0,3.1923505661,0.0722256678,-2.4901278192$ $0,0,-1.0719323079,-1.3161623366,0.3400112961$ $\mathrm{H}, 0,-1.0301562388,-2.0779060827,0.9022436438$ $\mathrm{C}, 0,-1.364881106,1.5905220442,0.3611921942$ $\mathrm{H}, \mathrm{O},-1.4157128749,1.6594184797,-0.7184326535$ $\mathrm{H}, \mathrm{O},-2.3758128392,1.5605431229,0.7568144535$ $\mathrm{H}, 0,-0.8808599536,2.4835087416,0.7462084785$

\begin{tabular}{|c|c|c|c|c|}
\hline $\mathrm{Si}$ & 0 & -0.43704 & -0.03971 & 0.42993 \\
\hline F & 0 & -0.37117 & -0.03299 & 2.08056 \\
\hline F & 0 & -0.21311 & 0.06776 & -1.45293 \\
\hline 0 & 0 & 1.29849 & 0.1229 & 0.40065 \\
\hline $\mathrm{H}$ & 0 & 2.00475 & 0.32176 & -0.78728 \\
\hline $\mathrm{H}$ & 0 & 1.64765 & 0.22432 & 1.27758 \\
\hline O & 0 & 2.0947 & 0.40967 & -1.83718 \\
\hline $\mathrm{H}$ & 0 & 1.09482 & 0.29884 & -1.99108 \\
\hline $\mathrm{H}$ & 0 & 2.56136 & -0.33865 & -2.20037 \\
\hline 0 & 0 & -1.33255 & 1.33301 & 0.32289 \\
\hline $\mathrm{H}$ & 0 & -1.53112 & 1.6093 & -0.55894 \\
\hline $\mathrm{C}$ & 0 & -1.16234 & -1.74684 & 0.18511 \\
\hline $\mathrm{H}$ & 0 & -1.89125 & -1.75484 & -0.6185 \\
\hline $\mathrm{H}$ & 0 & -0.38411 & -2.45901 & -0.0840 \\
\hline $\mathrm{H}$ & 0 & -1.63598 & -2.1024 & 1 \\
\hline
\end{tabular}

$\mathrm{SiMeF}(\mathrm{OH})_{2}-\mathrm{HF}\left(\mathrm{H}_{2} \mathrm{O}\right), \quad \mathrm{RC} 2$

Si, $0,-0.2380635759,-0.1829324858,0.9552758862$ $\mathrm{F}, 0,-0.1942295996,-0.1408301879,2.5466015063$ $0,0,1.3386054476,-0.1814763152,0.4424269163$ $\mathrm{H}, 0,1.9288658355,0.4222824494,0.8769093623$ $\mathrm{H}, 0,1.7700645485,-0.1958669722,-1.4646778236$ $0,0,1.68378375,0.0444114139,-2.3866039056$ $\mathrm{H}, 0,1.95932314,-0.6948427046,-2.9122713105$ $\mathrm{H}, 0,0.1712482718,0.811362214,-2.5255897411$ $\mathrm{F}, 0,-0.6774412009,1.1733416803,-2.3720720725$ $0,0,-1.0051687364,1.1745864037,0.5190697706$ $\mathrm{H}, \mathrm{O},-1.0823257349,1.3912196857,-0.4048208394$ C, $0,-1.0222285043,-1.7537458116,0.3621760748$ $\mathrm{H}, 0,-2.0414694036,-1.8316844997,0.7287695461$ $\mathrm{H}, 0,-1.0609375393,-1.7885687937,-0.7232119477$ $\mathrm{H}, \mathrm{O},-0.4712345143,-2.6231471587,0.7080667435$

$\operatorname{MeSiF}(\mathrm{OH})_{2}$ 
Si, $0,0.0079756281,0.0240379919,0.0119710388$

$\mathrm{F}, 0,0.0279559234,0.0041254527,1.6048350002$

$0,0,1.545543064,0.0509616419,-0.5394957173$

$\mathrm{H}, 0,2.1612946647,0.6887571091,-0.2059448291$

$\mathrm{C}, 0,-0.8159430091,-1.5048589892,-0.6337195563$

$\mathrm{H}, \mathrm{O},-0.8313025526,-1.5141889708,-1.7201661592$

$\mathrm{H}, \mathrm{O},-0.2895211095,-2.3955662981,-0.3044221986$

$\mathrm{H}, 0,-1.8418855122,-1.5684596079,-0.2835783362$

$0,0,-0.7827457245,1.4099647095,-0.3443394016$

$\mathrm{H}, \mathrm{O},-0.7685682569,1.7575399318,-1.2239997336$

$\operatorname{MeSiF}(\mathrm{OH})_{2}-\mathrm{H}_{2} \mathrm{O}, \mathrm{RC} 1$

Si, $0,-0.2487601452,0.4627010974,0.2084079224$

$\mathrm{F}, 0,-0.2130785806,0.3889050112,1.80799588$

$0,0,1.2412624861,0.4720516925,-0.4075718562$

$0,0,-0.9748445702,-0.9400623675,-0.2800360342$

$\mathrm{H}, 0,1.6536777537,-0.3559792131,-0.645131738$

$\mathrm{H}, 0,-1.7946218381,-1.192744353,0.1218700577$

$0,0,1.5179531223,-2.2857990589,-1.1136171324$

$\mathrm{H}, 0,0.5745652765,-2.3057548862,-0.9855107434$

$\mathrm{H}, 0,1.8783889171,-3.0470051299,-0.6794325624$

C, $0,-1.1796599404,1.9974669199,-0.2555942043$

$\mathrm{H}, 0,-0.6380048962,2.8816034032,0.0683000728$

$\mathrm{H}, 0,-1.3074195148,2.0618091682,-1.331873219$

$\mathrm{H}, \mathrm{O},-2.163245103,2.0257868979,0.2054697064$

$\operatorname{MeSiF}(\mathrm{OH})_{2}-\mathrm{H}_{2} \mathrm{O}$, TS

$\mathrm{F}, 0,0.0605132574,0.1715267951,-1.9046472411$

Si, $0,0.0477321805,0.1266442196,0.2721613963$

$0,0,1.657781079,0.1318953703,0.0478136272$

$0,0,-0.9115683283,1.415317911,0.0677103449$

$\mathrm{H}, 0,1.92543022,0.3179771141,-0.8442270362$

$0,0,-0.7078543884,-1.3713571057,-0.4852228485$

$\mathrm{H}, \mathrm{O},-0.5028690847,-1.0634026116,-1.4283088843$

$\mathrm{H}, \mathrm{O},-0.3308485057,-2.2201763658,-0.2743024114$

$\mathrm{H}, \mathrm{O},-0.9723923012,1.7220692468,-0.8289508814$

$\mathrm{C}, 0,-0.1687631591,-0.3571951117,2.0583148964$

$\mathrm{H}, 0,0.2145989011,0.4304437833,2.7008552924$

$\mathrm{H}, 0,-1.2160060232,-0.5021619826,2.3063607888$

$\mathrm{H}, 0,0.3749290056,-1.2651881497,2.3078403869$

$\operatorname{MeSi}(\mathrm{OH})_{3}-\mathrm{HF}, \mathrm{RC} 2$

Si, $0,-0.5116604832,-0.0117399481,-0.2722683544$

$0,0,-0.5223132487,-0.231911039,1.3405869567$

$0,0,1.1127706917,0.0264817011,-0.6715138231$

$\mathrm{H}, 0,2.2372394008,-0.0117105954,0.6813012458$

$\mathrm{H}, 0,0.2824337844,-0.2028835916,1.8440326022$

$\mathrm{H}, 0,1.3613597277,-0.0590064007,-1.582233419$

$\mathrm{F}, 0,2.5000098764,-0.0518448511,1.5711118585$

$0,0,-1.2355465378,-1.2669472558,-1.0302810759$

$\mathrm{H}, \mathrm{O},-1.1757837678,-2.1333547809,-0.651981989$ 
C, $0,-1.3697490945,1.5385845946,-0.8208584748$ $\mathrm{H}, 0,-2.4008242183,1.5433179345,-0.4798337463$ $\mathrm{H}, \mathrm{O},-0.8789908256,2.4207291304,-0.4202987103$ $\mathrm{H}, \mathrm{O},-1.3830688986,1.6213764186,-1.9044213608$

$\mathrm{SiMeF}(\mathrm{OH})_{2}-\left(\mathrm{H}_{2} \mathrm{O}\right)_{2}, \mathrm{RC} 1$

$\mathrm{Si}, 0,-0.3922932166,0.1852698566,0.9079874505$ $0,0,-0.3786699979,0.0334742045,2.5369485699$ $\mathrm{F}, 0,1.157243012,0.2864034713,0.4686085043$ $0,0,-1.0267835043,-1.1992087955,0.3254880423$ $\mathrm{H}, 0,-1.0017054306,-1.968976232,0.8782236726$ $\mathrm{H}, 0,0.2299273275,0.5256138803,3.0687650733$ $0,0,-0.5437840608,-0.3063469338,-2.5925969899$ $\mathrm{H}, 0,0.3828795109,-0.5221672636,-2.637555409$ $\mathrm{H}, \mathrm{O},-0.9393677428,-0.9754669813,-2.0487996079$ $0,0,2.343191049,-0.7256827978,-2.0615873352$ $\mathrm{H}, 0,2.2431087798,-0.3413242593,-1.1979254079$ $\mathrm{H}, 0,3.0350064133,-0.2481351737,-2.4989995382$ C, $0,-1.2745685624,1.66908493,0.2503376297$ $\mathrm{H}, 0,-1.2668520743,1.6581722568,-0.8343405553$ $\mathrm{H}, 0,-2.307503366,1.6803084414,0.5854876802$ $\mathrm{H}, 0,-0.8027920069,2.588171099,0.5877991713$

$\operatorname{SiMeF}(\mathrm{OH})_{2}-\left(\mathrm{H}_{2} \mathrm{O}\right)_{2}$, TS

$\mathrm{Si}, 0,-0.4567534684,-0.0014748801,0.4117962587$ $0,0,-0.4624661547,0.042309299,2.1105052355$ $0,0,1.2896260272,-0.0322786758,0.445423379$ $\mathrm{H}, 0,1.595928505,0.0532323543,1.3408719133$ $0,0,-1.2820682715,1.41814571,0.2167642432$ $\mathrm{H}, \mathrm{O},-1.4031278486,1.6833217689,-0.6829391266$ $\mathrm{H}, 0,-1.0676553785,0.6631969448,2.489723989$ $\mathrm{F}, 0,-0.1066322586,0.1457725636,-1.4994792551$ $\mathrm{H}, 0,2.0507557685,0.0696948248,-0.6653382393$ $0,0,2.2401860132,0.1567892242,-1.7224115696$ $\mathrm{H}, 0,1.2435867661,0.1923027776,-1.9394941812$ $\mathrm{H}, 0,2.6129033478,-0.6493120643,-2.0682042079$ $\mathrm{C}, 0,-1.2724860411,-1.6593645157,0.1055408746$ $\mathrm{H}, 0,-2.2102582882,-1.703941325,0.653198704$ $\mathrm{H}, 0,-1.4690969488,-1.868609827,-0.9387647531$ $\mathrm{H}, 0,-0.6461017057,-2.4547275696,0.5056160237$

$\operatorname{MeSi}(\mathrm{OH})_{3}$

Si, $0,0.0373473519,-0.0000811027,-0.004005359$ $0,0,0.0158652288,0.010875315,1.6413193591$ $\mathrm{H}, 0,0.8410382203,0.0133275343,2.1065847415$ $\mathrm{C}, 0,-1.7477840021,-0.0010875512,-0.5350029461$ $\mathrm{H}, 0,-2.2733185785,-0.87223452,-0.1522696373$ $\mathrm{H}, 0,-2.2708321731,0.8766692626,-0.1641471883$ $\mathrm{H}, 0,-1.8293945975,-0.0083424051,-1.618138725$ $0,0,0.8810574955,-1.2825826137,-0.5755981229$ $\mathrm{H}, \mathrm{O}, 0.6194049208,-2.1576017757,-0.3298288623$ 
$0,0,0.8846596436,1.2722720695,-0.5926933736$

$\mathrm{H}, 0,0.6242843505,2.1513244822,-0.3603305282$

$\mathrm{SiHF} \mathrm{OH}_{2}-\mathrm{SiHF}_{2} \mathrm{OH}, \mathrm{RC} 1$

Si, $0,2.397991854,-1.0403154254,-0.268659766$

$\mathrm{F}, 0,1.5385653123,-1.2603639821,1.063416573$

$0,0,1.3976309278,-0.8802285051,-1.5159199085$

$\mathrm{F}, 0,3.2227085917,0.2826916836,0.0118796968$

$\mathrm{H}, 0,3.3118723109,-2.1467104839,-0.4629299626$

$\mathrm{H}, 0,0.5726086067,-0.4180265857,-1.4005109952$

$0,0,-0.7536195287,0.2650841293,0.1592873328$

$\mathrm{H}, \mathrm{O},-0.3903756514,-0.2322330326,0.8839685248$

$\mathrm{Si}, 0,-2.1417723349,1.0942846569,0.3120475449$

$\mathrm{H}, 0,-1.979383527,2.4190971511,0.8801032225$

$\mathrm{F}, 0,-3.1490483474,0.2697155908,1.2121284222$

$\mathrm{F}, 0,-2.7515462453,1.2127854542,-1.1379801463$

SiHF $\mathrm{OH}_{2} \mathrm{SiHF}_{2} \mathrm{OH}$, homocondensation (TS)

Si, $0,-1.4376909152,0.3384338654,0.7909295371$

$\mathrm{F}, 0,-1.4656024141,0.7532322976,2.313978267$

$0,0,0.1432973408,-0.0022881548,0.4642369968$

$\mathrm{F}, 0,-2.2729041564,-0.9898692797,0.6561642469$

$\mathrm{H}, \mathrm{O},-1.968999552,1.3867383722,-0.0468360189$

$\mathrm{Si}, 0,1.1635254537,-0.3221842335,-1.0143266158$

$\mathrm{F}, 0,-0.2111484413,-0.2313129229,-1.8883197558$

$\mathrm{H}, 0,1.6054868189,-1.6152044801,-1.523504094$

$\mathrm{F}, 0,1.852691252,1.0001481727,-1.5593060303$

$0,0,2.2546596173,-0.422010375,0.4582665239$

$\mathrm{H}, 0,1.108719055,-0.1987261232,1.0098691966$

$\mathrm{H}, 0,2.7821283126,-1.1956987868,0.6053513014$

$\mathrm{SiHF}{ }_{3}-\mathrm{SiHF}_{2} \mathrm{OH}, \mathrm{RC} 1$

$\mathrm{Si}, 0,1.7437925944,-0.3600338998,-1.0071645822$ $\mathrm{F}, 0,1.4687477676,0.0654208171,0.4872969889$

$\mathrm{F}, 0,3.3108596144,-0.3654284572,-1.189382962$

$\mathrm{F}, 0,1.2555177334,-1.8427181222,-1.2095532356$

$\mathrm{H}, 0,1.1419169043,0.5468234034,-1.9551448688$

$0,0,-1.2718104476,-0.1667625199,-0.3987286266$

$\mathrm{H}, \mathrm{O},-1.9298809954,-0.3770269698,-1.0481996774$

$\mathrm{Si}, 0,-1.7234704249,0.4159589145,1.0511886328$

$\mathrm{H}, \mathrm{O},-1.0523467063,-0.2491604523,2.1455908927$

$\mathrm{F}, 0,-3.2963073854,0.2438157438,1.1734827643$

$\mathrm{F}, 0,-1.4354528406,1.9689671265,1.1194059954$

$\mathrm{SiHF}_{3}+\mathrm{SiHF}_{2} \mathrm{OH}$, heterocondensation (TS)

Si $, 0,-1.4131173983,-0.1239541986,0.8878520397$

$\mathrm{F}, 0,-1.348887523,-0.143143282,2.4612746906$

$0,0,0.1719545969,-0.0987500809,0.4022809863$

$\mathrm{F}, 0,-2.0460377819,-1.480950929,0.4099523175$

$\mathrm{H}, \mathrm{O},-2.1530800924,1.0134308446,0.396982529$ 
Si, $0,1.0847618626,0.1316782529,-1.09932451$

$\mathrm{F}, 0,-0.2834305909,0.3017411069,-1.9453686841$

$\mathrm{H}, \mathrm{0}, 1.7038402497,-0.9740334244,-1.7925456138$

$\mathrm{F}, 0,1.6641895233,1.5945993588,-1.2432619771$

$\mathrm{F}, 0,2.2880804923,-0.176498471,0.3471165153$

$\mathrm{H}, 0,1.2053534888,-0.2192635859,0.8705140198$

$\left(\mathrm{HF}_{2} \mathrm{Si}\right)_{2} \mathrm{O}$

Si, $0,0.9995066939,0.7323995411,-1.0195965219$

$\mathrm{F}, 0,0.3407396184,2.0903017948,-1.4858580721$

$0,0,0.0000124672,-0.0000668486,-0.000030136$

$\mathrm{F}, 0,1.1571586672,-0.1680808691,-2.3078358359$

$\mathrm{H}, 0,2.2987017052,0.9885463228,-0.4329417581$

$\mathrm{Si}, 0,-0.9993792287,-0.7327788428,1.019469049$

$\mathrm{F}, 0,-0.3395554268,-2.0897368609,1.4869745074$

$\mathrm{H}, 0,-2.2980003112,-0.990593374,0.4322688013$

$\mathrm{F}, 0,-1.1586301526,0.1683928312,2.3070192523$

$\left(\mathrm{HF}_{2} \mathrm{Si}\right){ }_{2} \mathrm{O}-\mathrm{H}_{2} \mathrm{O}, \mathrm{RC} 1$

Si, $0,-2.0287348762,-0.0309456081,0.6730481252$

$\mathrm{F}, 0,-2.0648779026,-0.0445255603,2.2539244137$

$0,0,-0.5058699247,-0.0336749924,0.186175157$

$\mathrm{F}, 0,-2.7263278881,-1.3664192062,0.1929747029$

$\mathrm{H}, 0,-2.7732880713,1.1107125507,0.1797908649$

$\mathrm{Si}, 0,1.0277541556,-0.0135111088,-0.3188691818$

$\mathrm{F}, 0,0.9815487155,0.0064222569,-1.9018543805$

$\mathrm{H}, 0,1.7481703505,-1.1516204118,0.1982824394$

$\mathrm{F}, 0,1.6435250403,1.3638105847,0.163090785$

$\mathrm{H}, 0,3.7796450413,0.0729085275,-2.1997394584$

$0,0,3.8289337705,0.042840495,-1.2529579764$

$\mathrm{H}, \mathrm{O}, 4.1698803165,0.8834766734,-0.9757961878$

$\left(\mathrm{HF}_{2} \mathrm{Si}\right)_{2} \mathrm{O}-\mathrm{H}_{2} \mathrm{O}$, TS

Si, $0,-1.3990800888,0.3763403041,0.2750911595$

$\mathrm{F}, 0,-1.8321599131,0.61501239,1.7996046355$

$0,0,0.1992682435,0.604285357,0.3494792216$

$\mathrm{F}, 0,-1.010853733,-0.5489978484,-1.5635108268$

$\mathrm{H}, \mathrm{0},-2.2533523869,1.2551506417,-0.4843493945$

$0,0,-1.8838338905,-1.3188596315,0.2468888906$

$\mathrm{H}, \mathrm{0},-1.4686682617,-1.4001072546,-0.7648386912$

$\mathrm{H}, 0,-1.630064495,-1.9218192719,0.9391537446$

$\mathrm{Si}, 0,1.5443783148,0.2973077174,-0.4968054213$

$\mathrm{H}, \mathrm{O}, 1.554352757,0.8268605685,-1.8426198098$

$\mathrm{F}, 0,2.74602174,0.9277118836,0.3158651601$

$\mathrm{F}, 0,1.7903343948,-1.2686778462,-0.4979913111$

$\mathrm{HF}_{2} \mathrm{SiOSiHF}(\mathrm{OH})$

Si, $0,-1.5363426239,0.014771562,0.4569356927$

$\mathrm{F}, 0,-1.5903586697,0.0722295036,2.045489689$

$0,0,0.0059157806,0.0299288703,-0.0032767443$ 
$\mathrm{H}, \mathrm{O},-2.2420463778,1.1834663873,-0.0441190947$

$\mathrm{Si}, 0,1.5267196127,-0.0264514163,-0.4948685476$

$\mathrm{F}, 0,1.5681375293,0.143891365,-2.0664897898$

$\mathrm{H}, 0,2.1928922912,-1.2576143618,-0.1196395288$

$\mathrm{F}, 0,2.3082765913,1.2061445431,0.1134083961$

$0,0,-2.1769257948,-1.3891928354,-0.0458865555$

$\mathrm{H}, \mathrm{O},-3.0225427002,-1.6886110496,0.256450334$ 
Table 1. Electronic energies and thermal corrections of model silanes (geometry optimization and vibrational analysis: $\mathrm{HF} / 6-31+\mathrm{G}^{*}$; final energies: B3LYP/6-311+G(2d,p)//HF/6-31+G*)

\begin{tabular}{|c|c|c|c|c|}
\hline species & $-\mathrm{E}_{0}$ & $\Delta \mathrm{H}^{298}$ & $\Delta \mathrm{G}^{298}$ & -E(final) \\
\hline $\mathrm{SiHF}_{3}$ & 588.03198 & 0.02245 & -0.00929 & 589.95611 \\
\hline $\mathrm{SiHF}_{2}(\mathrm{OH})$ & 564.01716 & 0.03359 & -0.00047 & 565.91941 \\
\hline $\mathrm{SiHF}(\mathrm{OH})_{2}$ & 539.99972 & 0.04484 & 0.00979 & 541.88010 \\
\hline $\mathrm{SiH}(\mathrm{OH})_{3}$ & 515.98070 & 0.05614 & 0.02014 & 517.83905 \\
\hline$\left(\mathrm{HF}_{2} \mathrm{Si}\right)_{2} \mathrm{O}$ & 1052.02496 & 0.04380 & -0.00234 & 1055.38072 \\
\hline $\mathrm{HF}_{2} \mathrm{SiOSiHF}(\mathrm{OH})$ & 1028.00789 & 0.05494 & 0.00738 & 1031.34180 \\
\hline $\mathrm{SiMeF}_{3}$ & 627.08744 & 0.05114 & 0.01376 & 629.30443 \\
\hline $\mathrm{SiMeF}_{2} \mathrm{OH}$ & 603.07192 & 0.06231 & 0.02374 & 605.26651 \\
\hline $\left.\mathrm{SiMeF}_{(\mathrm{OH}}\right)_{2}$ & 579.05159 & 0.07352 & 0.03382 & 581.22428 \\
\hline $\mathrm{SiMe}(\mathrm{OH})_{3}$ & 555.03018 & 0.08487 & 0.04390 & 557.18102 \\
\hline
\end{tabular}


Table 2. Electronic energies and thermal corrections of stationary points in hydrolysis of model silanes (geometry optimization and vibrational analysis: $\mathrm{HF} / 6-31+\mathrm{G}^{*}$; final energies: B3LYP/6-311+G(2d,p)//HF/6-31+G*)

\begin{tabular}{|c|c|c|c|c|c|}
\hline species & $-\mathrm{E}_{0}$ & $\Delta \mathrm{H}^{298}$ & $\Delta \mathrm{G}^{298}$ & -E(final) & BSSE \\
\hline $\mathrm{SiHF}_{3}-\mathrm{H}_{2} \mathrm{O}, \mathrm{RC} 1$ & 664.05733 & 0.04891 & 0.00673 & 666.42157 & 1.0 \\
\hline $\mathrm{SiHF}_{3}-\mathrm{H}_{2} \mathrm{O}, \mathrm{TS}$ & 663.99036 & 0.04447 & 0.00793 & 666.37446 & 2.1 \\
\hline $\mathrm{SiHF}_{2}(\mathrm{OH})-\mathrm{HF}, \mathrm{RC} 2$ & 664.04201 & 0.04806 & 0.00538 & 666.41210 & 0.6 \\
\hline $\mathrm{SiHF}_{3}-\left(\mathrm{H}_{2} \mathrm{O}\right)_{2}, \mathrm{RC} 1$ & 740.08727 & 0.07594 & 0.02568 & 742.89305 & 1.2 \\
\hline $\mathrm{SiHF}_{3}-\left(\mathrm{H}_{2} \mathrm{O}\right)_{2}, \mathrm{TS}$ & 740.04249 & 0.07382 & 0.03289 & 742.86492 & 2.6 \\
\hline $\mathrm{SiHF}_{2}(\mathrm{OH})-\mathrm{HF}\left(\mathrm{H}_{2} \mathrm{O}\right), \mathrm{RC} 2$ & 740.07189 & 0.07511 & 0.02222 & 742.88415 & \\
\hline $\mathrm{SiHF}_{2}(\mathrm{OH})-\mathrm{H}_{2} \mathrm{O}, \mathrm{RC} 1 \mathrm{a}$ & 640.04772 & 0.06056 & 0.01543 & 642.39087 & 0.8 \\
\hline $\mathrm{SiHF}_{2}(\mathrm{OH})-\mathrm{H}_{2} \mathrm{O}, \mathrm{RC} 1$ & 640.04058 & 0.06008 & 0.01627 & 642.38287 & 0.8 \\
\hline $\mathrm{SiHF}_{2}(\mathrm{OH})-\mathrm{H}_{2} \mathrm{O}, \mathrm{TS}$ & 639.97955 & 0.05666 & 0.01975 & 642.34108 & \\
\hline $\mathrm{SiHF}(\mathrm{OH})_{2}-\mathrm{HF}, \mathrm{RC} 2$ & 640.02784 & 0.05949 & 0.01790 & 642.37698 & \\
\hline $\mathrm{SiHF}_{2}(\mathrm{OH})-\left(\mathrm{H}_{2} \mathrm{O}\right)_{2}, \mathrm{RC} 1 \mathrm{a}$ & 716.07100 & 0.08750 & 0.03735 & 718.85522 & \\
\hline $\mathrm{SiHF}_{2}(\mathrm{OH})-\left(\mathrm{H}_{2} \mathrm{O}\right)_{2}, \mathrm{RC} 1$ & 716.07011 & 0.08715 & 0.03550 & 718.85394 & \\
\hline $\mathrm{SiHF}_{2}(\mathrm{OH})-\left(\mathrm{H}_{2} \mathrm{O}\right)_{2}, \mathrm{TS}$ & 716.02409 & 0.08501 & 0.04365 & 718.82670 & \\
\hline $\mathrm{SiHF}(\mathrm{OH})_{2}-\mathrm{HF}\left(\mathrm{H}_{2} \mathrm{O}\right), \mathrm{RC} 2$ & 716.06182 & 0.08674 & 0.03764 & 718.85350 & \\
\hline $\mathrm{SiHF}(\mathrm{OH})_{2}-\mathrm{H}_{2} \mathrm{O}, \mathrm{RC} 1 \mathrm{a}$ & 616.02989 & 0.07202 & 0.02847 & 618.35179 & \\
\hline $\mathrm{SiHF}(\mathrm{OH})_{2}-\mathrm{H}_{2} \mathrm{O}, \mathrm{RC} 1$ & 616.02831 & 0.07198 & 0.02822 & 618.34962 & \\
\hline $\mathrm{SiHF}(\mathrm{OH})_{2}-\mathrm{H}_{2} \mathrm{O}, \mathrm{TS}$ & 615.96173 & 0.07017 & 0.03303 & 618.29930 & \\
\hline $\mathrm{SiH}(\mathrm{OH})_{3}-\mathrm{HF}, \mathrm{RC} 2$ & 616.01063 & 0.07083 & 0.02831 & 618.33776 & \\
\hline $\mathrm{SiHF}(\mathrm{OH})_{2}-\left(\mathrm{H}_{2} \mathrm{O}\right)_{2}, \mathrm{RC} 1$ & 692.05170 & 0.09859 & 0.04616 & 694.81372 & 0.9 \\
\hline $\mathrm{SiHF}(\mathrm{OH})_{2}-\left(\mathrm{H}_{2} \mathrm{O}\right)_{2}$, TS & 692.00062 & 0.09588 & 0.05339 & 694.78315 & \\
\hline $\mathrm{SiHF}(\mathrm{OH})_{2}-\mathrm{HF}\left(\mathrm{H}_{2} \mathrm{O}\right), \mathrm{RC} 2$ & 692.04565 & & & 694.81512 & \\
\hline$\left(\mathrm{SiHF}_{2} \mathrm{OH}\right)_{2}, \mathrm{RC} 1$ & 1128.04260 & 0.06966 & 0.01389 & 1131.84644 & \\
\hline$\left(\mathrm{SiHF}_{2} \mathrm{OH}\right)_{2}, \mathrm{TS}$ & 1127.97444 & 0.06558 & 0.01721 & 1131.79374 & \\
\hline $\mathrm{SiHF}_{3}-\mathrm{SiHF}_{2} \mathrm{OH}, \mathrm{RC} 1$ & 1152.05283 & 0.05803 & 0.00322 & 1155.87801 & \\
\hline $\mathrm{SiHF}_{3}-\mathrm{SiHF}_{2} \mathrm{OH}, \mathrm{TS}$ & 1151.98780 & 0.05378 & 0.00609 & 1155.82917 & \\
\hline$\left(\mathrm{HF}_{2} \mathrm{Si}\right)_{2} \mathrm{O}-\mathrm{H}_{2} \mathrm{O}, \mathrm{RC} 1$ & 1128.05087 & 0.07027 & 0.01573 & 1131.84701 & \\
\hline$\left(\mathrm{HF}_{2} \mathrm{Si}\right)_{2} \mathrm{O}-\mathrm{H}_{2} \mathrm{O}, \mathrm{TS}$ & 1127.98387 & 0.06618 & 0.01823 & 1131.80170 & \\
\hline $\mathrm{SiMeF}_{3}-\mathrm{H}_{2} \mathrm{O}, \mathrm{RC} 1$ & 703.11172 & 0.07763 & 0.03037 & 705.76883 & 0.8 \\
\hline $\mathrm{SiMeF}_{3}-\mathrm{H}_{2} \mathrm{O}, \mathrm{TS}$ & 703.04354 & 0.07339 & 0.03240 & 705.71998 & \\
\hline $\mathrm{SiMeF}_{2} \mathrm{OH}-\mathrm{HF}, \mathrm{RC} 2$ & 703.09812 & 0.07684 & 0.02981 & 705.76095 & \\
\hline $\mathrm{SiMeF}_{3}-\left(\mathrm{H}_{2} \mathrm{O}\right)_{2}, \mathrm{RC} 1$ & 779.14128 & 0.10463 & 0.04901 & 782.23988 & 0.9 \\
\hline $\mathrm{SiMeF}_{3}-\left(\mathrm{H}_{2} \mathrm{O}\right)_{2}, \mathrm{TS}$ & 779.09105 & 0.10233 & 0.05676 & 782.20644 & 2.7 \\
\hline $\mathrm{SiMeF}_{2} \mathrm{OH}-\mathrm{HF}\left(\mathrm{H}_{2} \mathrm{O}\right), \mathrm{RC} 2$ & 779.12829 & 0.10391 & 0.04837 & 782.23351 & 1.3 \\
\hline $\mathrm{SiMeF}_{2} \mathrm{OH}-\mathrm{H}_{2} \mathrm{O}, \mathrm{RC} 1$ & 679.10189 & 0.08936 & 0.03973 & 681.73697 & 0.8 \\
\hline $\mathrm{SiMeF}_{2} \mathrm{OH}-\mathrm{H}_{2} \mathrm{O}, \mathrm{TS}$ & 679.03253 & 0.09442 & 0.05422 & 681.68565 & \\
\hline
\end{tabular}




\begin{tabular}{|c|c|c|c|c|c|}
\hline species & $-\mathrm{E}_{0}$ & $\Delta \mathrm{H}^{298}$ & $\Delta \mathrm{G}^{298}$ & $-\mathrm{E}($ final $)$ & BSSE \\
\hline $\mathrm{SiMeF}(\mathrm{OH})_{2}-\mathrm{HF}, \mathrm{RC} 2$ & 679.08192 & 0.08825 & 0.04215 & 681.72334 & \\
\hline $\mathrm{SiMeF}_{2} \mathrm{OH}-\left(\mathrm{H}_{2} \mathrm{O}\right)_{2}, \mathrm{RC} 1$ & 755.12444 & 0.11599 & 0.05947 & 758.20100 & 0.9 \\
\hline $\mathrm{SiMeF}_{2} \mathrm{OH}-\left(\mathrm{H}_{2} \mathrm{O}\right)_{2}$, TS & 755.07236 & 0.11341 & 0.06742 & 758.16777 & \\
\hline $\mathrm{SiMeF}(\mathrm{OH})_{2}-\mathrm{HF}\left(\mathrm{H}_{2} \mathrm{O}\right), \mathrm{RC} 2$ & 755.11526 & & & 758.19905 & \\
\hline $\mathrm{SiMeF}(\mathrm{OH})_{2}-\mathrm{H}_{2} \mathrm{O}, \mathrm{RC} 1$ & 655.08310 & 0.10079 & 0.05299 & 657.69706 & 0.8 \\
\hline $\mathrm{SiMeF}(\mathrm{OH})_{2}-\mathrm{H}_{2} \mathrm{O}, \mathrm{TS}$ & 655.01310 & 0.09947 & 0.05826 & 657.64166 & \\
\hline $\mathrm{SiMe}(\mathrm{OH})_{3}-\mathrm{HF}, \mathrm{RC} 2$ & 655.06375 & 0.09959 & 0.05245 & 657.68279 & \\
\hline $\mathrm{SiMeF}(\mathrm{OH})_{2}-\left(\mathrm{H}_{2} \mathrm{O}\right)_{2}, \mathrm{RC} 1$ & 731.10571 & 0.12729 & 0.06964 & 734.16051 & 0.9 \\
\hline $\mathrm{SiMeF}(\mathrm{OH})_{2}-\left(\mathrm{H}_{2} \mathrm{O}\right)_{2}$, TS & 731.04825 & 0.12428 & 0.07663 & 734.12355 & \\
\hline $\mathrm{SiMe}(\mathrm{OH})_{3}-\mathrm{HF}\left(\mathrm{H}_{2} \mathrm{O}\right), \mathrm{RC} 2$ & 731.09821 & 0.12692 & 0.07295 & 734.15952 & \\
\hline
\end{tabular}

Table 3. Electronic energies and thermal corrections of stationary points in hydrolysis of model silanes in water (geometry optimization and vibrational analysis: $\mathrm{SCRF} / \mathrm{HF} / 6-31+\mathrm{G}^{*}$; final energies: SCRF/B3LYP/6-311+G(2d,p)//HF/6-31+G*; model IEF-PCM, UAHF)

\begin{tabular}{|c|c|c|c|c|}
\hline species & $-\mathrm{E}_{0}$ & $\Delta \mathrm{H}^{298}$ & $\Delta \mathrm{G}^{298}$ & $-E($ final $)$ \\
\hline $\mathrm{SiHF}_{3}$ & 588.03618 & 0.02218 & -0.00969 & 589.95939 \\
\hline $\mathrm{SiHF}_{3}-\mathrm{H}_{2} \mathrm{O}, \mathrm{RC} 1$ & 664.07098 & 0.04785 & 0.00547 & 666.43432 \\
\hline $\mathrm{SiHF}_{3}-\mathrm{H}_{2} \mathrm{O}, \mathrm{TS}$ & 664.00933 & 0.04314 & 0.00592 & 666.39175 \\
\hline $\mathrm{SiHF}_{2}(\mathrm{OH})$ & 564.02989 & 0.03260 & -0.00144 & 565.93105 \\
\hline $\mathrm{SiHF}_{2}(\mathrm{OH})-\mathrm{H}_{2} \mathrm{O}, \mathrm{RC} 1$ & 640.06469 & 0.05830 & 0.01671 & 642.40753 \\
\hline $\mathrm{SiHF}_{2}(\mathrm{OH})-\mathrm{H}_{2} \mathrm{O}, \mathrm{TS}$ & 640.00400 & 0.05497 & 0.01728 & 642.36241 \\
\hline $\mathrm{SiHF}(\mathrm{OH})_{2}$ & 540 & 0.0 & 0.00 & 840 \\
\hline $\mathrm{SiHF}(\mathrm{OH})_{2}-\mathrm{H}_{2} \mathrm{O}, \mathrm{RC} 1$ & 616.05371 & 0.06889 & 0.02577 & 618.37405 \\
\hline $\mathrm{SiHF}(\mathrm{OH})_{2}-\mathrm{H}_{2} \mathrm{O}, \mathrm{TS}$ & 615.99147 & 0.06808 & 0.02999 & 618.32397 \\
\hline $\mathrm{SiH}(\mathrm{OH})_{3}$ & 516.00594 & 0.05398 & 0.01773 & 517.86255 \\
\hline$\left(\mathrm{HF}_{2} \mathrm{Si}\right)_{2} \mathrm{O}$ & 1052.03152 & 0.04333 & -0.00214 & 1055.38581 \\
\hline
\end{tabular}

